\title{
Measurements of Heavy Quark Production at DØ
}

\author{
L. Markosky \\ University of Arizona \\ Tucson, Arizona
}

Fermi National Accelerator Laboratory

P.O. Box 500, Batavia, Illinois 60510

\section{May 1995}

Presented at the XXXth Rencontres de Moriond on QCD and High Energy Interactions, Les Arcs, France, March 19-26, 1995. 


\section{Disclaimer}

This report was prepared as an account of work sponsored by an agency of the United States Government. Neither the United States Government nor any agency thereof, nor any of their employees, makes any warranty, express or implied, or assumes any legal liability or responsibility for the accuracy, completeness, or usefulness of any information, apparatus, product, or process disclosed, or represents that its use would not infringe privately owned rights. Reference herein to any specific commercial product, process, or service by trade name, trademark, manufacturer, or otherwise, does not necessarily constitute or imply its endorsement, recommendation, or favoring by the United States Government or any agency thereof. The views and opinions of authors expressed herein do not necessarily state or reflect those of the United States Government or any agency thereof. 


\title{
Measurements of Heavy Quark Production at Do
}

\author{
L. Markosky $\nmid \ddagger$ \\ $\dagger$ University of Arizona, \\ Tucson, Arizona, USA
}

For the D0 Collaboration at Fermilab

\begin{abstract}
We report measurements of the inclusive single muon and $b$-quark cross sections in $p \bar{p}$ collisions at $\sqrt{s}=1.8 \mathrm{TeV}$ using the $\mathrm{D} 0$ detector at the Fermilab collider, for the kinematic range $\left|y_{b}\right|<1.0$ and $p_{t}^{b}>6 \mathrm{GeV} / \mathrm{c}$. We also report preliminary measurements on the $J / \psi$ and $\Upsilon$ production cross sections.
\end{abstract}

\section{Introduction}

The D0 experiment at the Fermilab Tevatron has measured the inclusive single muon cross section for $\left|\eta_{\mu}\right|<0.8$ in $p \bar{p}$ collisions at $\sqrt{s}=1.8 \mathrm{TeV}$ using data taken during the $1992-93$ collider run. The b-quark cross section is extracted using these measurements and found to be in agreement, within experimental error and theoretical uncertainty, with next-to-leading order QCD predictions, though all of the data are systematically high with respect to the prediction. The inclusive $J / \psi$ and $\Upsilon$ cross sections in the central region have also been measured. These results are similarly high with respect to theoretical expectation.

The D0 detector has been described elsewhere [1]. It consists of inner tracking chambers used to measure the primary vertex and to help identify electrons and muons, uranium-liquid argon calorimeters to detect electrons, photons and jets, and iron toroids and drift chambers to detect muons. The combined calorimeter plus toroid thickness varies from about $14 \lambda$ in the central region to $19 \lambda$ in the end. This thickness reduces the punchthrough backgrounds to less than $1 \%$ of prompt muon production and also allows for clean muon identification within hadronic jets. Muon momentum is measured using the toroid and has a

† E-mail: Leigh@fnaldo.fnal.gov resolution of $\sigma(p) / p=0.18(p-2) / 2 \oplus 0.008 p$ (where $p$ is in $\mathrm{GeV} / \mathrm{c}$ ).

\section{The $b$ Quark Cross Section Analysis}

We have measured b-quark production cross section using the semileptonic decays $b \rightarrow \mu+X$. For this paper, only results from the central region with $\left|\eta_{\mu}\right|<0.8$ are presented. Events were collected using a single muon trigger which required a coincidence in the level 0 beambeam scintillation counters which indicated that an inelastic collision had occured. The level 1 muon trigger uses $60 \mathrm{~cm}$ wide hodoscopic elements formed from the muon drift chambers, and required 2 or 3 layers of chambers to have hits consistent with a muon produced in the interaction region. The minimum energy of muons exiting the toroid is about $3.2 \mathrm{GeV}$ and the level 1 muon trigger became fully efficient at $5 \mathrm{GeV}$. At level 2 , muons are identified using a software filter composed of the initial part of the offline reconstruction. The single muon trigger requires one or more muons at level 1 and level 2, while the dimuon trigger used in the $J / \psi$ and $\Upsilon$ analyses requires two or more at each level.

Good muons were selected offline by requiring each muon to have hits in all three layers of the muon system for the single muon cross section analyses (this requirement is relaxed by one for the dimuon cross 
section). Additionally each muon was required to have at least $1 \mathrm{GeV}$ of associated calorimeter energy, possess a matching track in the central tracking chamber, point back to the interaction vertex in the bend and nonbend views, and traverse a minimum field integral of $0.6 \mathrm{GeV} / \mathrm{c}$ in the toroids.

All muons were required to have $\left|\eta^{\mu}\right|<0.8$. Additional kinematic cuts applied were $p_{t}^{\mu}>3.5 \mathrm{GeV} / \mathrm{c}$ for the single muon analysis, and $p_{t}^{\mu}>3 \mathrm{GeV} / c$ for the dimuons. The $J / \psi$ analysis requires that all dimuons have invariant mass $M_{\mu \mu}<6 \mathrm{GeV} / c^{2}$ while the $\Upsilon$ requires invariant mass $>6 \mathrm{GeV} / c^{2}$. In addition, the $\Upsilon$ analysis makes a cut on the 3-space opening angle between the muons to remove cosmic ray background, $\Phi_{3 d}<160^{\circ}$, and requires that each muon appear isolated in the calorimeter. The isolation parameter, $I$, is defined as the difference between the energy observed in the calorimeter in a cone of $R=0.2$ in $\eta-\phi$ space about the muon, minus the expected energy, divided by the error on that expectation:

$$
I=\frac{E_{\text {observed }}-E_{\text {expected }}}{\sigma_{e}}
$$

For these analyses, residual cosmic ray contamination was estimated by fitting the crossing time distribution in the muon chambers (called floating TO) to distributions of known beam produced muons and cosmic rays. In all analyses the remaining cosmic ray contamination is approximately $10 \%$. After all cuts, the number of single muon events is 16000 from $73 \mathrm{nb}^{-1}$ of data. For the $\Upsilon$ and $J / \psi$ analyses, there were 540 events from 7.3 $p b^{-1}$, and 1740 events in $6.6 p b^{-1}$, respectively.

Trigger and offline selection efficiencies were determined by passing Monte Carlo events through the full D0 detector, trigger simulation, and reconstruction packages. In all cases the efficiencies were cross-checked using appropriate data samples.

The resulting single muon cross section is shown in Figure 1 compared to expectation for contribution from $\pi / K$ and $W / Z$ decays from ISAJET. The excess in the data compared to the prediction for these sources we attribute to muons from the decay of heavy $(b / c)$ quarks. The fraction of the inclusive muon spectrum originating from $b$-quark decay was estimated by fitting the distributions of muon $p_{t}$ relative to the nearest reconstructed jet axis $\left(p_{t}^{\text {rel }}\right)$ with the $p_{t}^{\text {rel }}$ shapes predicted by ISAJET for muons from the decays of $b, c$, and $\pi / K$ sources. $p_{t}^{\text {rel }}$ is a quantity which depends upon the $Q$ value of the decay, and is relatively insensitive to the details of the particular Monte Carlo involved. About $60 \%$ of the events in the inclusive single muon sample reconstuct a jet of $E_{t}>8 \mathrm{GeV}$ within a cone of $R=0.7$ in $\eta-\phi$ space about the muon. This fraction is consistent with predictions from simulations, and all kinematic distributions for the samples with

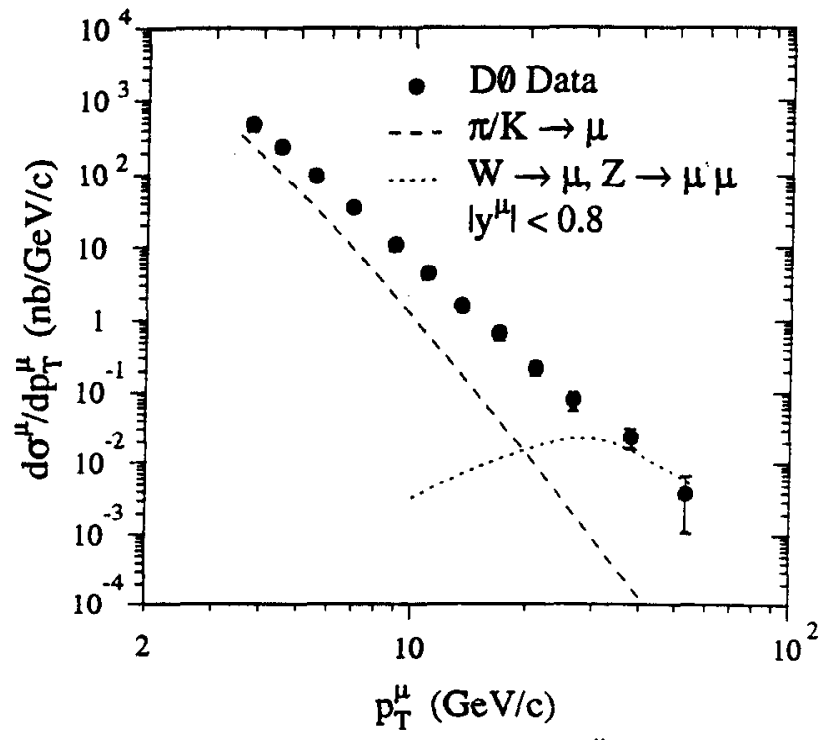

Figure 1. The single muon cross section vs. $p_{t}^{\mu}$ for muons with $|\eta|<0.8$.

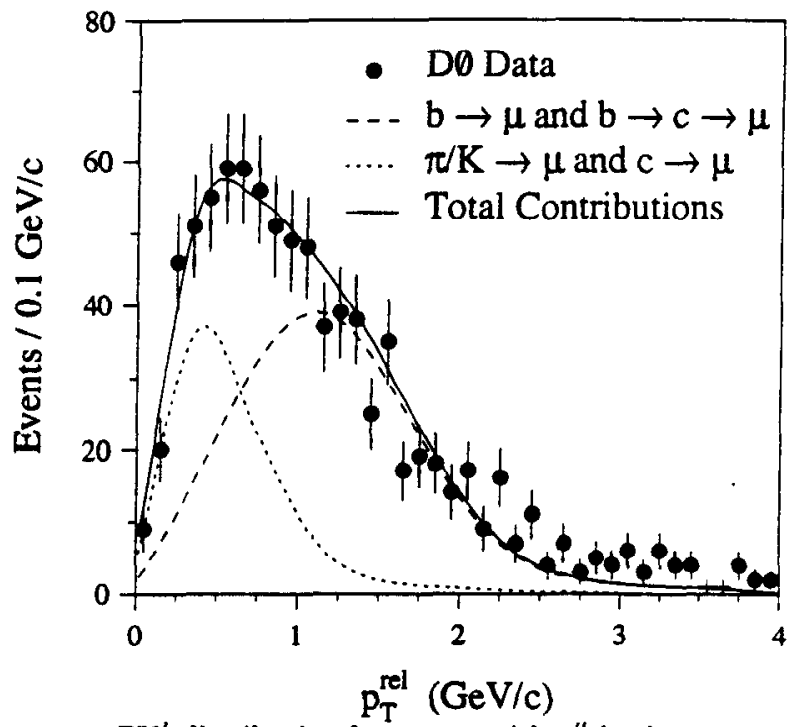

FIgure 2. $P_{t}^{r e l}$ distribution for muons with $p_{t}^{\mu}$ in the range $8<p_{t}<30 \mathrm{GeV} / \mathrm{c}$, and $\left|\eta^{\mu}\right|<0.8$.

and without recontructed jets have been checked to be similar. The $b$ fraction extracted from this method is thus assumed to hold for the entire sample. The result of one of the $p_{t}^{\text {rel }}$ fits to a single bin of the inclusive muon $p_{t}$ spectrum of Figure 1 is shown in Figure 2. The results of the $p_{t}^{\text {rel }}$ fits to each of the bins of the inclusive spectrum is shown in Figure 3. The fitted points are compared to the $b$ fraction extracted by calculating the $b$ fraction directly, assuming the $b / c$ ratio present in ISAJET. The two methods give consistent results, predicting a $b$ fraction of about $25 \%$ for muons with $p_{t}^{\prime \prime} \simeq 5 \mathrm{GeV} / \mathrm{c}$, rising to about $80 \%$ for muons with $p_{t}^{\mu}>15 \mathrm{GeV} / \mathrm{c}$. For both methods, the contribution from $W / Z$ decay was calculated directly using the Do measured cross sections, and subtracted from the data prior to the fitting procedure.

Once the fractions of the single muon inclusive cross sections coming from b-quark decay is determined, 


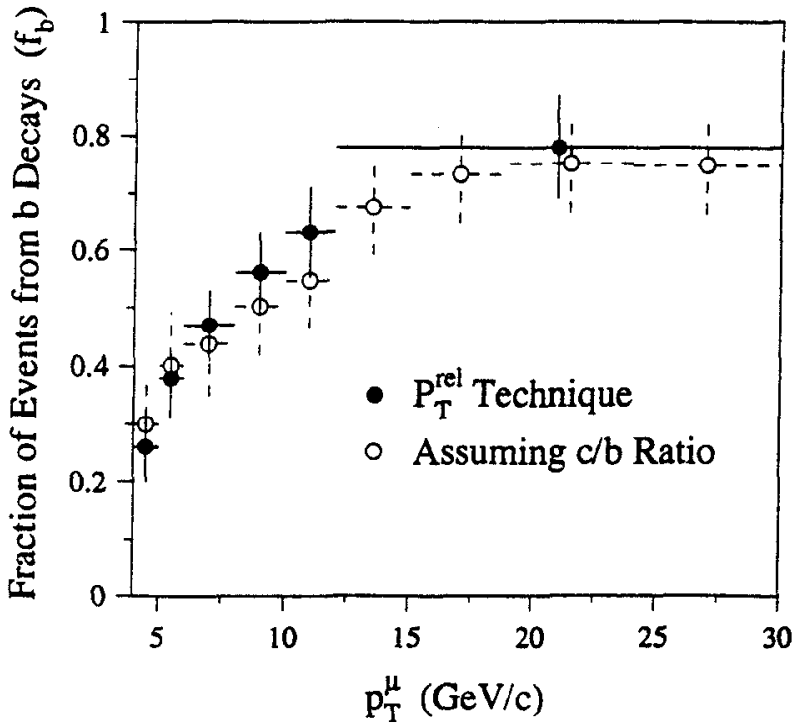

Figure 3. $f_{b}$ as a function of $p_{t}^{\mu}$ : the solid points are from the $P_{t}^{r e l}$ fitting technique and the open circles are from the $b / c$ ratio method.

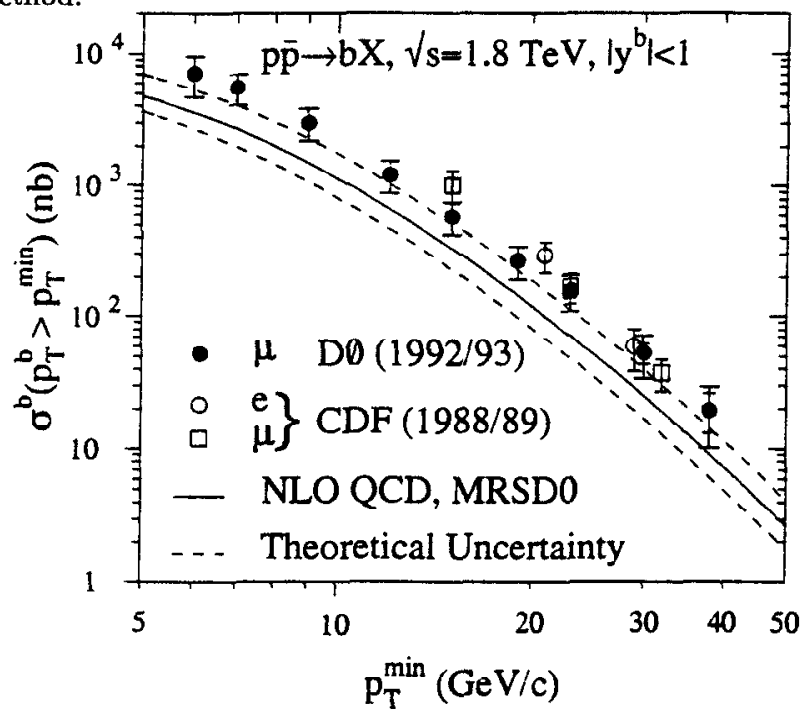

Figure 4. The $b$-quark cross section vs. $k_{t}^{\min }$ for the single muon analysis.

the integrated inclusive $b$-quark cross section can be extracted following the method of UA1 and CDF in order to facilitate comparison $[2,3]$. The resulting $b$ quark cross section is plotted in Figure 4 The results are consistent with the next-to-leading order (NLO) QCD calculations of Nason et al. [4], within experimental errors and theoretical uncertainty, though all of the data points are systematically high relative to the prediction. the range of theoretical uncertainty shown in Figure 4 is obtained by varying the renormalization $\mu$ and the $\Lambda$ parameter within the range $\mu_{0} / 2<\mu<2 \mu_{0}$ and $100<\Lambda<187 \mathrm{MeV}$.

\section{Inclusive $J / \psi$ Cross Section}

To select $J / \psi$ candidates, each muon was required to pass similar track quality criteria as in the dimuon

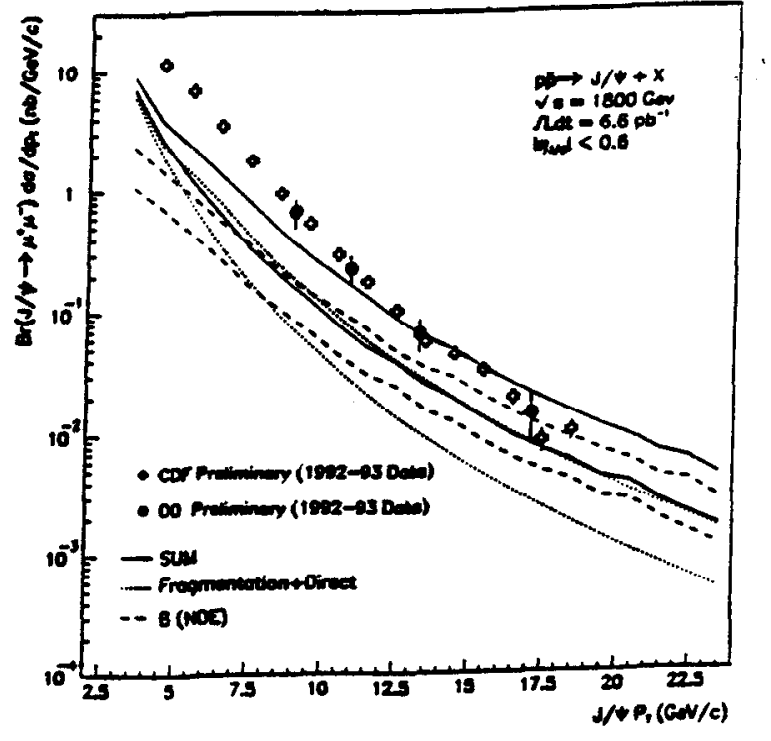

Figure 5. Inclusive $J / \psi$ cross section a function of $P_{t}^{J / \psi}$.

cross section analysis described above. The dimuon was required to have oppositely signed muons, and $\left|\eta_{w}\right|<$ 0.6. The number of $J / \psi$ after all cuts was estimated by fitting the invariant dimuon mass distribution to the sum of a Gaussian centered at the $J / \psi$ mass and a polynomial background (consisting mostly of sequential $b$-quark decays). The total number of $J / \psi$ given by the fit was $414 \pm 50$ from data runs having a total integrated luminosity of $6.6 \mathrm{pb}^{-1}$. The overall $J / \psi$ detection efficiency is a combination of trigger and offline efficiencies, and was determined using complete detector and trigger simulations. The number of $J / \psi$ 's in each $p_{t}^{\psi}$ bin was determined from the fit described above. The inclusive cross section times branching ratio shown in Figure 5 was obtained by dividing the data bin by bin by the $J / \psi$ detection efficiency and the integrated luminosity. The D0 data points are in good agreement with the CDF data [5]. Also shown are the theoretical estimates for $J / \psi$ production from $b$-quark decay, and direct charmonium production [6] plus fragmentation processes. The measured inclusive $\psi$ cross section is in reasonable agreement with the predicted contribution for the sum of these three sources at higher values of $P_{t}^{J / \psi}$, but there is considerable excess in the data at lower values of $P_{t}^{J / \psi}$.

\subsection{Fraction of $J / \psi$ from Direct Charmonium.}

$J / \psi$ 's from direct charmonium production arise primarily through the decay of $\chi_{c}$. The muons from $\chi_{c}$ are expected to be isolated, and are accompanied by a low $E_{t}$ photon. Events with photons were tagged by requiring the presence in the event of an electromagnetic 


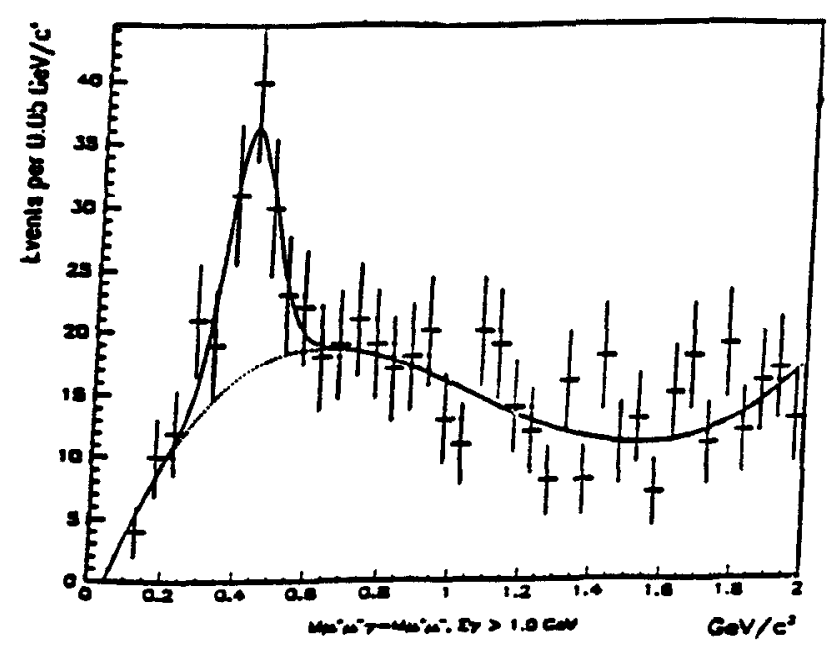

Figure 6. $M_{\mu \mu \gamma}-M_{\mu \mu}$ for the $\chi_{c} \longrightarrow J / \psi X$ sample.

calorimeter cluster of energy $E_{\gamma}>0.6 \mathrm{GeV}$. Figure 6 shows the distribution of mass difference, $M^{\mu \mu \gamma}-M^{\mu \mu}$, with a peak at $0.43 \mathrm{GeV} / \mathrm{c}^{2}$. The number of $\chi_{c}$ 's predicted from the fit (after background subtraction) is $66 \pm 15 \pm 5$. Correcting this number by the photon detection efficiency and isolation efficiency gives a fraction of $J / \psi$ with $P_{t}^{J / \psi}>8 \mathrm{GeV} / \mathrm{c}$ and $|\eta|<0.6$ from $\chi_{c}$ decay equal to $0.30 \pm 0.07 \pm 0.10$, and for $P_{t}^{J / \psi}>10 \mathrm{GeV} / \mathrm{c}$, $|\eta|<0.6$, of $0.22 \pm 0.07 \pm 0.09$.

\section{Inclusive Upsilon Cross Section}

The data sample for the $\Upsilon$ analysis was selected from the same data as for the dimuon analyses described above. Since muons from $\Upsilon$ decay are expected to be isolated, at least one of the muons in each event was required to satisfy an isolation cut defined in equation 1. A simultaneous maximum likelihood fit to three distributions: dimuon mass, energy deposition in a wide cone of $R=0.6$ minus the energy in cone of $R=0.2$ about the muon (a quantity termed muon "halo"), and floating To (defined earlier in this note), was used to extract the number of $\Upsilon$ 's from the background (QCD, Drell-Yan, and cosmic rays). The shapes used in the fit were derived from appropriate data samples, and in the case of the mass distribution, from Monte Carlo. The result of this fit is shown in Figure 7. The number of $\Upsilon$ 's predicted in the sample from this fit was $84 \pm 11$. Table 4 shows the predictions from the fit for the numbers of events from the different sources contributing to the total sample.

\begin{tabular}{|l||c|}
\hline Contribution & \# of events \\
\hline$\Upsilon$ & $84 \pm 11$ \\
Cosmics & $7 \pm 5$ \\
QCD & $115 \pm 14$ \\
Drell-Yan & $33 \pm 10$ \\
\hline
\end{tabular}

Correcting $N_{\Upsilon}$ for the acceptance, the offline cuts, and the trigger efficiency, and the integrated luminosity of the sample, the total cross section times branching fraction to muons was calculated to be:

$$
\begin{gathered}
\sigma_{\Upsilon} \cdot B R\left(P_{t}(\Upsilon)>1 \mathrm{GeV} / \mathrm{c},|\mathrm{y}|<0.7\right)= \\
1.15 \pm 0.13 \text { (stat) } \pm 0.25 \text { (sys)nb }
\end{gathered}
$$

To obtain a total cross section, a total branching fraction for the $1 S, 2 S$ and $3 S$ states (which DO does not resolve) was calculated using Particle Data Group branching fractions [7], together with preliminary CDF cross sections $[8]$ for these states:

$$
\begin{aligned}
& B R(1 S, 2 S, 3 S)= \\
& \frac{\sigma(1 S) \cdot B R(1 S)+\sigma(2 S) \cdot B R(2 S)+\sigma(3 S) \cdot B R(3 S)}{\sigma(1 S, 2 S, 3 S)}
\end{aligned}
$$

Bearing in mind that this total branching fraction is based upon cross sections which are themselves preliminary, the total $\Upsilon$ production cross section calculated in this manner is calculated to be

$$
\begin{gathered}
\sigma_{\Upsilon}\left(P_{t}(\Upsilon)>1 \mathrm{GeV} / \mathrm{c},|\mathrm{y}|<0.7\right)= \\
57 \pm 6.2 \text { (stat) } \pm 12.5(\text { sys }) \mathrm{nb}
\end{gathered}
$$

\section{Conclusions}

The b-quark cross section measured by $D 0$ in the region $\left|y_{b}\right|<1$ is in agreement, within the range of experimental error and theoretical uncertainty, with the next-to-leading order QCD prediction [4]. However, the data points are systematically high with respect to that prediction. The inclusive $J / \psi$ and $\Upsilon$ cross sections in the regions $\left|y_{\psi}\right|<0.6$ and $\left|y_{\Upsilon}\right|<0.7$ are also high respect to prediction $[6,9]$.

\section{References}

[1] S. Abachi et al., Nucl. Instrum. \& Methods A338 (1994) 185.

[2] C. Albajar et al. Phys. Lett. B186 (1987) 237. 

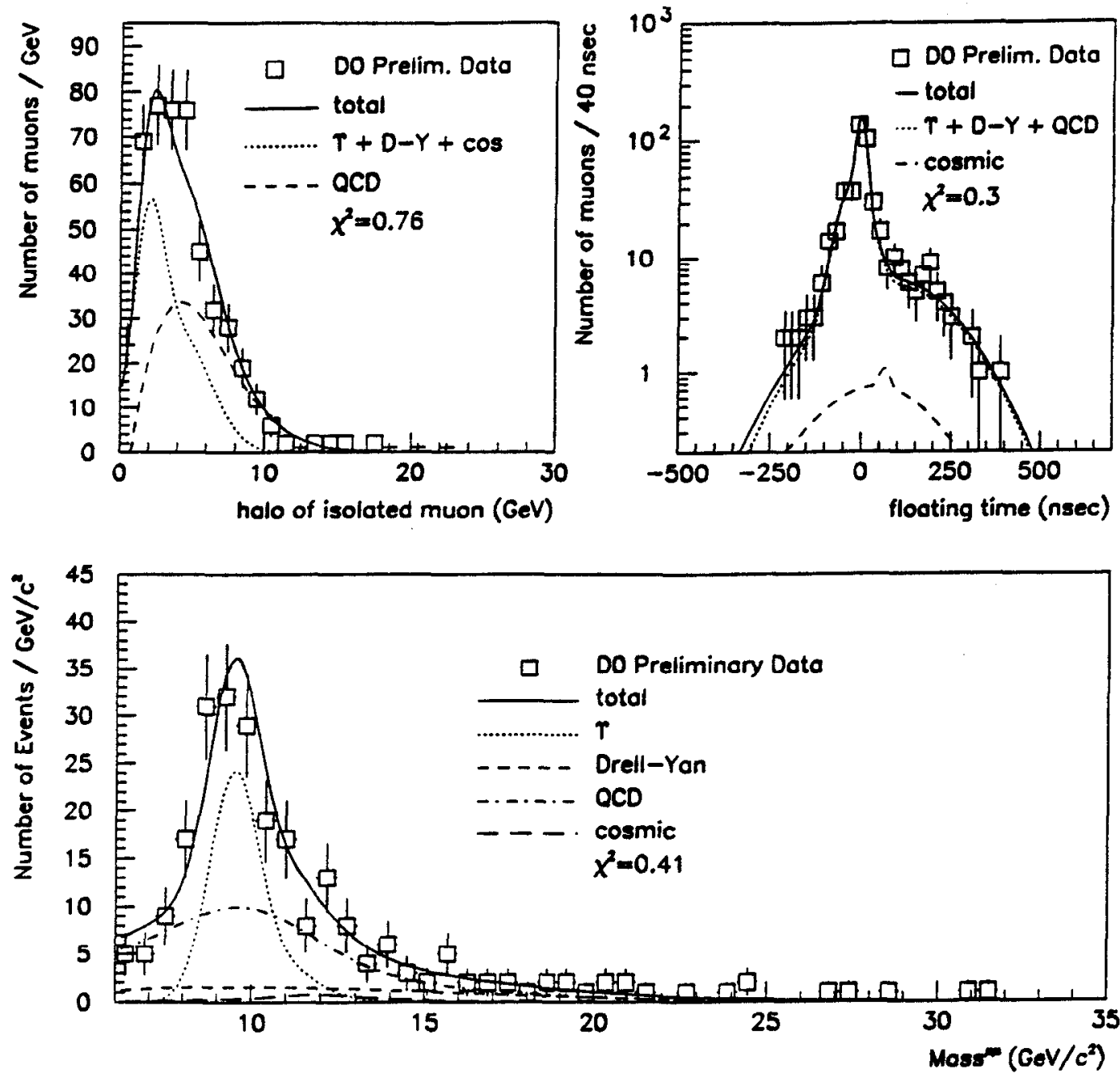

Figure 7. Result of simultaneous fit to the mass, To, and Halo distributions in the $T$ sample.

[3] F. Abe et al., Phys. Rev. Lett. 71 (1993) 2396.

[4] P. Nason, S. Dawson, and R.K. Ellis, Nucl. Phys. B303 (1988) 607; B327 (1989) 49; B335 (1990) 260.

[5] F. Abe et al. Phys. Rev. Lett. 69 (1992) 3704.

[6] M. Mangano, private communication.

[7] Review of Particle Properties, Phys. Rev. $\mathbf{D 4 5}$ (1995).

[8] V. Papadimitriou, Proceedings of the 1994 DPF Meeting , 2-6 Aug., 1994, Albuquerque, NM (to be published).
[9] V. Barger, A. Martin, Phys. Rev. D, 31 (1985) 1051 . 